\title{
PREVALÊNCIA DA SÍNDROME METABÓLICA E DE SEUS COMPONENTES EM PESSOAS COM DIABETES MELLITUS TIPO 2
}

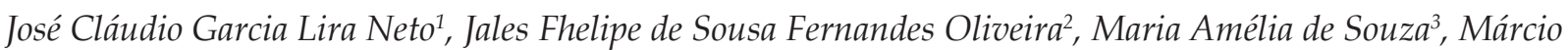 \\ Flávio Moura de Araújo ${ }^{4}$, Marta Maria Coelho Damasceno ${ }^{5}$, Roberto Wagner Júnior Freire de Freitas ${ }^{6}$
}

\footnotetext{
${ }^{1}$ Mestre em Ciências e Saúde. Docente do Departamento de Enfermagem da Universidade Federal do Piauí (UFPI). Floriano, Piauí, Brasil. E-mail: jclira@live.com

2 Enfermeiro. UFPI. Floriano, Piauí, Brasil. E-mail: jales_fhelipe@hotmail.com

${ }^{3}$ Doutora em Enfermagem. Docente do Departamento de Enfermagem da Universidade Federal de Pernambuco. Vitória de Santo Antão, Pernambuco, Brasil. E-mail: souza_mariaamelia@hotmail.com

${ }^{4}$ Doutor em Enfermagem. Docente do Curso de Enfermagem da Universidade da Integração Internacional da Lusofonia AfroBrasileira. Redenção, Ceará, Brasil. E-mail: marciofma@unilab.edu.br

${ }^{5}$ Doutora em Enfermagem. Docente do Programa de Pós-Graduação em Enfermagem da Universidade Federal do Ceará. Fortaleza, Ceará, Brasil. E-mail: marta.damasceno@pq.cnpq.br

${ }^{6}$ Doutor em Enfermagem. Pesquisador em Saúde Pública da Fundação Oswaldo Cruz. Fortaleza, Ceará, Brasil. E-mail: robertowjff@ gmail.com
}

\section{RESUMO}

Objetivo: identificar a prevalência da Síndrome Metabólica e seus componentes em pessoas com Diabetes Mellitus tipo 2.

Método: estudo transversal com 201 pessoas cadastradas e acompanhadas em Unidades de Saúde. Foram investigadas variáveis sociodemográficas, antropométricas, clínicas e às relacionadas à Síndrome Metabólica. A coleta de dados foi realizada nas Unidades Básicas de Saúde, durante a consulta de enfermagem.

Resultados: a prevalência da Síndrome Metabólica de 46,3\%, significativamente associada ao sexo feminino ( $p=0,001)$ e às pessoas com excesso de peso $(\mathrm{p}=0,001)$. Quando os componentes da síndrome foram analisados de forma isolada, as taxas de circunferência da cintura, glicemia de jejum, triglicerídeos e pressão arterial, se mostraram elevados em $62,0 \%, 58,5 \%, 55,8 \%$ e 49,8\%, respectivamente. Já a taxa de HDL se mostrou baixa em $47,3 \%$.

Conclusão: com 46,3\% dos investigados apresentando Síndrome Metabólica, atenção especial deve ser dada às mulheres e pessoas com excesso de peso, pois além de apresentarem percentuais significativamente maiores de Síndrome Metabólica, também apresentaram alterações nos principais componentes.

DESCRITORES: Síndrome X metabólica. Diabetes Mellitus Tipo 2. Doença crônica. Saúde do adulto. Enfermagem. Marcadores clínicos. Atenção primária à saúde.

\section{PREVALENCE OF THE METABOLIC SYNDROME AND ITS COMPONENTS IN PEOPLE WITH TYPE 2 DIABETES MELLITUS}

\footnotetext{
ABSTRACT

Objective: to identify the prevalence of the Metabolic Syndrome and its components in people with Type 2 Diabetes Mellitus.

Method: cross-sectional study with 201 people enrolled and monitored in Health Units. Sociodemographic, anthropometric, clinical variables and those related to the Metabolic Syndrome were investigated. The data collection was carried out at the Basic Health Units, during the nursing consultation.

Results: the prevalence of the Metabolic Syndrome was 46.3\%, significantly associated with the female gender ( $p=0.001)$ and overweight people $(\mathrm{p}=0.001)$. When the components of the syndrome were analyzed in isolation, the waist circumference, fasting glycemia, triglycerides and blood pressure were $62.0 \%, 58.5 \%, 55.8 \%$ and $49.8 \%$, respectively. And the HDL rate was low, in $47.3 \%$.

Conclusion: having $46.3 \%$ of those investigated with the Metabolic Syndrome, special attention should be paid to women and overweight individuals, since in addition to presenting significantly higher percentages of Metabolic Syndrome, they have also presented changes in the main components.

DESCRIPTORS: Syndrome X metabolic. Type 2 Diabetes Mellitus. Chronic disease. Adult's health. Nursing. Clinical markers. Primary health care.
} 


\section{PREVALENCIA DEL SÍNDROME METABÓLICO Y SUS COMPONENTES EN PERSONAS CON DIABETES MELLITUS TIPO 2}

\section{RESUMEN}

Objetivo: identificar la prevalencia del Síndrome Metabólico y sus componentes en personas con Diabetes Mellitus tipo 2.

Método: estudio transversal con 201 personas registradas y acompañadas en Unidades de Salud. Fueron investigadas las variables sociodemográficas, antropométricas, clínicas y las relacionadas con el Síndrome Metabólico. La recolección de datos fue realizada en las Unidades Básicas de Salud durante la consulta de enfermería.

Resultados: la prevalencia del Síndrome Metabólico de $46,3 \%$ es significativamente asociado con el sexo femenino (p=0,001) y las personas con exceso de peso $(\mathrm{p}=0,001)$. Cuando los componentes del síndrome fueron analizados de forma aislada, las tasas de circunferencia de la cintura, glicemia de ayuno, triglicéridos y presión arterial se mostraron elevadas en $62,0 \%, 58,5 \%, 55,8 \%$ y $49,8 \%$, respectivamente. Sin embargo, la tasa de HDL se mostró baja, con un $47,3 \%$.

Conclusión: con el 46,3\% de los investigados presentando el Síndrome Metabólico, debe darse una atención especial para las mujeres y personas con exceso de peso porque además de presentar porcentajes significativamente mayores del Síndrome Metabólico, también presentaron alteraciones en los principales componentes.

DESCRIPTORES: Síndrome metabólico. Diabetes Mellitus Tipo 2. Enfermedad crónica. Salud del adulto. Enfermerías. Marcadores clínicos. Atención primaria para la salud.

\section{INTRODUÇÃO}

A síndrome metabólica (SM) pode ser definida como um complexo compilado de distúrbios metabólicos, acompanhado de alto risco para o desenvolvimento de Diabetes Mellitus tipo 2 (DM2) e de doenças cardiovasculares (DCV), caracterizado por hiperglicemia, hipertensão, níveis elevados de triglicerídeos (TG), valores diminuídos de colesterol de alta densidade (HDL-c), além da obesidade abdominal. Sua prevalência já atinge cerca de um quarto da população adulta mundial e é responsável por aumentar em duas vezes o risco de morte e em até cinco vezes o risco para desenvolvimento de DM2. ${ }^{1-2}$

Estudos conduzidos na América do Sul demonstraram altos valores de prevalência, variando de $12,3 \%$ a $44,6 \%$, dependendo do critério utilizado para o diagnóstico. Particularizando para o Brasil, estudo de prevalência conduzido em um estado do Nordeste mostrou que a SM esteve presente em 50,7\% de adultos com DM2, causando danos econômicos e sociais inestimáveis, pela agregação de fatores e irregularidades cardiometabólicas. ${ }^{3-5}$ Nesse contexto, salienta-se que a predisposição genética, a resistência insulínica, a obesidade abdominal, a inatividade física, uma dieta inadequada, a presença de estados pró-inflamatórios e as alterações hormonais compõem o rol das possíveis causas relacionadas ao aparecimento da SM, havendo, ainda, uma direta relação com os diferentes grupos éticos espalhados pelo mundo. ${ }^{1}$

Para o diagnóstico da SM, segundo o critério International Diabetes Federation (IDF), o indivíduo deve apresentar circunferência abdominal eleva$\mathrm{da}$, adicionado de, pelo menos, dois dos seguintes componentes: TG $\geq 150 \mathrm{mg} / \mathrm{dL}$; HDL-c $<40 \mathrm{mg} / \mathrm{dL}$ (homens) ou $<50 \mathrm{mg} / \mathrm{dL}$ (mulheres); Pressão arterial (PA) $\geq 130 / 85 \mathrm{mmHg}$ ou uso de anti-hipertensivos; e,
Glicemia de jejum (GJ) $\geq 100 \mathrm{mg} / \mathrm{dL}$ ou diagnóstico prévio de DM2. ${ }^{6}$

Levando em consideração a magnitude da $\mathrm{SM}$ e os potenciais agravos que ela pode provocar, fica perceptível que as mais distintas populações devem ser investigadas, sejam crianças, adolescentes, adultos e ou idosos. Entretanto, um dos grupos que merece destaque são as pessoas com DM2. O diabetes não só atinge mais de 400 milhões de pessoas em todo o mundo, sendo 14,3 milhões delas no Brasil, mas também, mostra-se tímida em produções científicas que objetivam identificar a prevalência da SM nessa população, principalmente em se tratando de investigações realizadas no nordeste brasileiro, especialmente, envolvendo a enfermagem. ${ }^{7}$ Além disso, não há um consenso no que se refere ao critério diagnóstico utilizado para identificar a presença da SM. ${ }^{5}$

Nessa direção, estudos epidemiológicos devem ser estimulados para que preencham as lacunas no conhecimento dos cenários em que se encontra a SM, na coexistência com outras enfermidades, como o DM2, visando à construção de intervenções fundamentadas em programas interdisciplinares que estimulem à mudança do estilo de vida, contribuindo para a redução da incidência de complicações atribuídas a estas patologias. Dessa forma, o objetivo desta investigação consistiu em identificar a prevalência da SM e de seus componentes, em pessoas com DM2, por meio do critério da IDF.

\section{MÉTODO}

Estudo transversal, desenvolvido em 17 Unidades Básicas de Saúde (UBS) na cidade de Floriano, Piauí. A população do estudo foi composta por pessoas com diagnóstico médico de DM2, cadastradas e 
acompanhadas nas unidades, no período de agosto de 2014 a abril de 2015.

No período estimado, 1.124 pessoas com DM2, maiores de 18 anos, eram acompanhadas nas UBS da cidade. Dessa forma, inicialmente, mais de 500 indivíduos com DM2 foram recrutados para participar da pesquisa. Para serem inclusos no estudo, os participantes deveriam ser maiores de 18 anos, diagnosticados com DM2 e em tratamento com antidiabéticos orais há, no mínimo, seis meses. Foram excluídos da pesquisa pessoas em uso combinado de antidiabéticos orais e insulina, gestantes e pacientes hospitalizados, uma vez que essas condições trariam alterações às taxas metabólicas dos participantes analisados. Assim, após a aplicação dos critérios estabelecidos, 412 pessoas foram selecionadas.

No entanto, durante a coleta dos dados, apenas 201 pessoas compareceram nos dias e horários pré-estabelecidos. Mesmo assim, a amostra foi representativa para a população investigada. Cabe ressaltar que após a coleta dos exames sanguíneos, algumas amostras foram insuficientes para a análise dos parâmetros metabólicos, necessitando de uma segunda coleta. Todavia, alguns participantes recusaram-se a colaborar com essa fase, causando alguns missings na amostra final.

Foi utilizado um formulário para captação de dados sociodemográficos (idade, sexo, estado civil e laboral, classe econômica, entre outros), antropométricos e clínicos (altura, peso, estado nutricional, prática de exercício físico, uso de tabaco e consumo de álcool) e referentes à análise da $\mathrm{SM}$, a saber: circunferência da cintura (CC), PA, TG, HDL-c e GJ.

Para a classificação da SM, conforme o critério da IDF, os participantes do estudo deveriam ter, pelo menos, dois componentes adicionais à CC elevada ( $\geq 90 \mathrm{~cm}$ para homens $\mathrm{e} \geq 80 \mathrm{~cm}$ para mulheres), a saber: PA ( $\geq 130 / 85 \mathrm{~mm} / \mathrm{Hg}$ ou uso de anti-hipertensivo); HDL-c ( $<40 \mathrm{mg} /$ dl para homens e $<50 \mathrm{mg} /$ dl para mulheres); TG $(\geq 150 \mathrm{mg} / \mathrm{dl}$ ) e ou glicemia de jejum GJ ( $\geq 100 \mathrm{mg} / \mathrm{dl}$ ou diagnóstico prévio para DM2). ${ }^{6}$ Para a captação das amostras bioquímicas de TG, GJ e HDL-c, os participantes do estudo se submeteram a um jejum alimentar de 12 horas. Os dados foram categorizados em "normal", "elevado" ou "diminuído".

A coleta de dados ocorreu durante as consultas de enfermagem aos pacientes com DM2 nas UBS. Todas as etapas da coleta de dados foram executadas por acadêmicos de enfermagem e enfermeiros previamente capacitados, visando assegurar uma boa qualidade na operacionalização e padronização dos dados coletados. Após a explicação dos objetivos do estudo, os participantes eram esclarecidos sobre os possíveis riscos e benefícios oriundos da pesquisa, bem como orientados sobre a necessidade da realização de exames laboratoriais.

Os dados antropométricos (peso e altura) foram avaliados uma única vez, mediante alguns cuidados. O peso foi obtido com os participantes descalços e com roupas leves, por meio do uso de uma balança portátil digital com capacidade para $150 \mathrm{~kg}$ e uma precisão de $0,1 \mathrm{~kg}$. A estatura foi verificada a partir de uma fita métrica com escala de $0,5 \mathrm{~cm}$. A fim de assegurar a precisão da medida, os pesquisados foram orientados a se posicionarem eretos e imóveis, com as mãos espalmadas sobre as coxas e com a cabeça ajustada ao plano de Frankfurt. Já o estado nutricional foi calculado através do Índice de Massa Corporal (IMC), definido como a razão entre o peso $(\mathrm{kg})$ e o quadrado da altura $(\mathrm{m})$. Foram considerados com sobrepeso, os participantes com valores situados entre 25,0 e $29,9 \mathrm{~kg} / \mathrm{m}^{2}$; e com obesidade, àqueles com IMC $\geq 30 \mathrm{~kg} / \mathrm{m}^{2}$. $^{6-7}$

As informações sobre o uso de tabaco e álcool foi autorreferido. No que se refere ao tabaco, os participantes que afirmaram fazer uso da droga foram considerados tabagistas e classificados em fumantes diários (que fumam pelo menos um cigarro por dia) ou fumantes ocasionais). Para o consumo de álcool, foi utilizado o Single Question Alcohol Screening Test. Por sua vez, os participantes classificados como sedentários foram aqueles que praticaram atividades físicas por um tempo menor do que 150 minutos semanais. ${ }^{7}$ Para a aferição da PA foram utilizados esfigmomanômetros aneróides da marca Tycos ${ }^{\circledast}$ e manguitos da marca Welch Allyn ${ }^{\circledR}$, de diferentes tamanhos, com a largura da borracha correspondente a $40 \%$ da circunferência do braço e o comprimento envolvendo pelo menos $80 \%$. Foram utilizados, ainda, estetoscópios biauriculares da marca Littmann ${ }^{\circledR}$ para técnica auscultatória. Foram tomadas três medidas, com intervalo mínimo de um minuto entre cada uma, e a média das duas últimas mensurações foi computada como o valor da PA..$^{5-7}$

Os valores obtidos foram digitados em uma planilha no Excel ${ }^{\circledR}$, versão 2010, e em seguida exportados para análise no software estatístico livre Epi-Info ${ }^{\circledR}$, versão 3.5.3. Inicialmente, foram calculadas medidas de tendência central. Na análise da normalidade das variáveis foi adotado o teste de Bartlett. Com base nessa informação, foram empregados testes paramétricos (Teste T) ou não paramétricos (Teste Kruskal-Wallis). Na associação das proporções de variáveis, foi empregado teste do Qui-quadrado e Teste exato de Fisher, no caso de tabelas $2 \times 2$. Em todas as análises, foi adotado um intervalo de confiança de 95\% e nível de significância de 0,05. 
A pesquisa foi submetida e aprovada pelo Comitê de Ética em Pesquisa com Seres Humanos da Universidade Federal do Piauí sob o parecer $n^{\circ}$ 485.420/2013 e CAAE: 07054412.6.0000.5214. Somente após a anuência do entrevistado e assinatura do Termo de Consentimento Livre e Esclarecido (TCLE) iniciou-se a coleta dos dados, respeitando todos os preceitos éticos estabelecidos na Resolução nº 466/12.

\section{RESULTADOS}

Dos 201 participantes investigados com DM2 prevaleceram na amostra as mulheres $(72,6 \%)$ e pessoas com baixa escolaridade $(29,4 \%$ de analfabetos / ensino fundamental incompleto), sendo encontrada uma média de 4,7 anos de estudo (DP $\pm 4,34)$. A idade variou de 19 a 96 anos, com média de 63,1 anos $(\mathrm{DP} \pm 12,5)$. Predominaram, ainda, as pessoas que se autorreferiram pardas (68,7\%); aposentadas (50,7\%), tendo como renda principal a própria aposentadoria $(44,5 \%)$; em união estável $(50,2 \%)$; seguidoras de alguma religião $(95,0 \%)$; que não faziam uso de álcool $(87,6 \%)$ ou tabaco $(89,1 \%)$; com excesso de peso $(71,6 \%)$; e, sedentárias $(71,1 \%)$.

Quanto à distribuição da prevalência dos componentes da SM, a tabela 1 demonstra que $62,0 \%$ dos participantes encontravam-se com a CC elevada, (componente considerado pré-requisito para o diagnóstico da síndrome segundo a IDF). A prevalência da SM detectada na presente população foi de $46,3 \%$.

Tabela 1 - Distribuição da prevalência dos componentes da síndrome metabólica segundo o critério International Diabetes Federation. Floriano, PI, Brasil, 2015. (n=201)

\begin{tabular}{lcc}
\hline \multicolumn{1}{c}{ Variáveis } & $\mathbf{n}^{*}$ & $\%$ \\
\hline Circunferência da cintura $(\mathrm{n}=200)$ & & \\
Normal & 76 & 38,0 \\
Elevada & 124 & 62,0 \\
Glicemia de jejum (n=164) & & \\
Normal & 68 & 41,5 \\
Elevada & 96 & 58,5 \\
HDL-c† (n=148) & & \\
Normal & 78 & 52,7 \\
Diminuído & 70 & 47,3 \\
Triglicerídeos (n=165) & \\
Normal & 73 & 44,2 \\
Elevado & 92 & 55,8 \\
Pressão arterial (n=201) & & \\
Normal & 101 & 50,2 \\
Elevada & 100 & 49,8 \\
\hline *n: amostra; † HDL-c: High density lipoprotein-cholesterol.
\end{tabular}

${ }^{*} \mathrm{n}$ : amostra; † HDL-c: High density lipoprotein-cholesterol.
A tabela 2 faz uma associação entre a SM e as variáveis sociodemográficas, antropométricas e clínicas. É possível identificar que o sexo feminino $(p=0,001)$ e as pessoas com excesso de peso $(p=0,001)$ apresentaram, significativamente, maiores percentuais de SM.

Tabela 2 - Associação da síndrome metabólica com as variáveis sociodemográficas, antropométricas e clínicas. Floriano, PI, Brasil, 2015. (n=201)

\begin{tabular}{|c|c|c|c|c|c|}
\hline \multirow{3}{*}{ Variáveis } & \multicolumn{5}{|c|}{ Síndrome Metabólica } \\
\hline & \multicolumn{2}{|c|}{ Sim } & \multicolumn{2}{|c|}{ Não } & \multirow[b]{2}{*}{$p$} \\
\hline & $\mathbf{n}^{*}$ & $\%$ & $\mathbf{n}$ & $\%$ & \\
\hline Sexo & & & & & $0,001 \dagger$ \\
\hline Feminino & 79 & 54,1 & 67 & 45,9 & \\
\hline Masculino & 14 & 25,5 & 41 & 74,5 & \\
\hline Cor & & & & & $0,835 \dagger$ \\
\hline Branca & 9 & 40,9 & 13 & 59,1 & \\
\hline Negra & 20 & 48,8 & 21 & 51,2 & \\
\hline Parda & 64 & 46,4 & 74 & 53,6 & \\
\hline Situação conjugal & & & & & $0,363+$ \\
\hline Casado/união estável & 45 & 44,6 & 56 & 55,4 & \\
\hline Solteiro, viúvo ou divorciado & 48 & 48,0 & 52 & 52,0 & \\
\hline Situação laboral & & & & & $0,905 \dagger$ \\
\hline $\begin{array}{l}\text { Possui emprego (formal/ } \\
\text { informal) }\end{array}$ & 15 & 42,9 & 20 & 57,1 & \\
\hline Aposentado & 48 & 47,1 & 54 & 52,9 & \\
\hline Desempregado/ do lar & 30 & 46,9 & 34 & 53,1 & \\
\hline Escolaridade & & & & & $0,901 \dagger$ \\
\hline Analfabeto & 28 & 47,5 & 31 & 52,5 & \\
\hline Ensino fundamental & 50 & 46,7 & 57 & 53,3 & \\
\hline Ensino médio/superior & 15 & 42,9 & 20 & 57,1 & \\
\hline Religião & & & & & $0,529+$ \\
\hline Possui religião & 88 & 46,1 & 103 & 53,9 & \\
\hline Não tem religião & 5 & 50,0 & 5 & 50,0 & \\
\hline IMC & & & & & $0,001 \dagger$ \\
\hline Eutrófico & 3 & 5,3 & 54 & 94,7 & \\
\hline Sobrepeso & 51 & 65,4 & 27 & 34,6 & \\
\hline Obesidade & 39 & 59,1 & 27 & 40,9 & \\
\hline Tabagismo & & & & & $0,381 \dagger$ \\
\hline Sim & 9 & 40,9 & 13 & 59,1 & \\
\hline Não & 84 & 46,9 & 95 & 53,1 & \\
\hline Etilismo & & & & & $0,093+$ \\
\hline Sim & 8 & 32,0 & 17 & 68,0 & \\
\hline Não & 85 & 48,3 & 91 & 51,7 & \\
\hline Sedentarismo & & & & & $0,417 \dagger$ \\
\hline Sim & 28 & 48,3 & 30 & 51,7 & \\
\hline Não & 65 & 45,5 & 78 & 54,5 & \\
\hline
\end{tabular}

Cada um dos cinco componentes da SM, também, foi associado às variáveis sociodemográficas, antropométricas e clínicas. A circunferência da cin- 
tura elevada esteve presente em maiores percentuais nas mulheres $(\mathrm{p}=0,001)$ e naqueles com sobrepeso/ obesidade $(p=0,001)$. Os níveis de TG aumentados estiveram associados ao sexo feminino $(p=0,002)$ e aos participantes tabagistas $(p=0,041)$. O HDL-c esteve com seus valores reduzidos nas mulheres $(p=0,006)$ e nos participantes obesos $(p=0,029)$. A glicemia venosa de jejum alterada esteve associada aos participantes que faziam uso de álcool $(p=0,043)$. A pressão arterial elevada esteve estatisticamente associada aos participantes com baixo nível de escolaridade $(\mathrm{p}=0,005)$, aos com excesso de peso $(p=0,005)$ e tabagistas $(p=0,021)$.

\section{DISCUSSÃO}

A prevalência encontrada de SM na presente investigação foi de $46,3 \%$. As buscas por estudos que, também, utilizaram o critério da IDF para identificar SM em brasileiros, revelaram percentuais distintos, variando de $35,7 \%$ a 43,2\%. ${ }^{8-9}$ Ao comparar tais achados com pesquisas internacionais, ${ }^{10-14}$ verifica-se que as prevalências encontradas no Brasil se mostram, ligeiramente, maiores.

No entanto, a literatura não é unânime, e diferentes resultados estão diretamente associados aos hábitos culturais e características étnicas dos locais de investigação. A exemplo, estudos conduzidos no México, Estados Unidos, Colômbia, Hungria, Irã, Brasil e Gana, mostraram taxas de prevalência da SM de $68,7 \%, 51 \%, 41 \%, 38 \%, 34,6 \%, 32 \%$ e $29,2 \%$, respectivamente. ${ }^{15-21}$

Cabe ressaltar que, devido às características multiculturais e a variabilidade demográfica e epidemiológica da população brasileira, torna-se difícil generalizar os resultados nacionais. O que se pode destacar é que pesquisas brasileiras envolvendo a prevalência de SM em pacientes com DM2 por meio do critério da IDF ainda são tímidas, impossibilitando maiores comparações de resultados.

Nessa pesquisa observou-se a predominância do sexo feminino. Tal fato pode estar relacionado, principalmente, à maior preocupação das mulheres com relação à saúde, bem como ao baixo comparecimento dos homens aos serviços de atenção primária à saúde, o que repercute no desenvolvimento de complicações crônicas das doenças, em especial o DM2, na sobrecarga da atenção secundária e terciária e na elevação dos custos aos cofres públicos e ao sistema de saúde. ${ }^{22}$ Entretanto, este estudo constatou que a SM foi significativamente maior nas mulheres $(p=0,001)$, corroborando com os achados na literatura. ${ }^{1,22-24}$
Estudo desenvolvido no Sudeste brasileiro, com objetivo de identificar SM em pessoas com diabetes residentes da zona urbana e rural da cidade de Coimbra, Minas Gerais, revelou que o público feminino é três vezes mais propenso à síndrome que os homens. ${ }^{25} \mathrm{Tal}$ fato pode ser justificado, uma vez que as mulheres apresentam maiores alterações na circunferência da cintura (fator condicionante para a SM), seja pela própria fisiologia e deposição de gordura localizada, seja pela inadequação dos hábitos alimentares e sedentarismo. ${ }^{1}$

Quanto à idade, estudos que investigaram a SM em diferentes populações mostraram que a prevalência dessa síndrome se encontra mais presente em pessoas maiores de 50 anos de idade, indo ao encontro da pesquisa em tela. ${ }^{14,22,25}$

Alterações cognitivas e funcionais, que progridem com o avançar dos anos, interferem na capacidade de compreensão das informações sobre a doença, gerando um déficit no autocuidado. ${ }^{1,10,21}$ Outro possível fator que predispõe ao aparecimento da SM é a baixa escolaridade. ${ }^{1-5}$ Nessa investigação, houve um registro de baixa escolaridade entre os participantes, ficando a média de estudos em 4,7 anos. Esse dado implica de forma negativa no conhecimento sobre o DM2 e sobre a SM, tendo por consequência, um acúmulo de fatores de risco e desordens que progridem para futuras incapacidades ou óbitos. ${ }^{5}$

Ainda que sempre lembrados durantes as consultas de enfermagem e ações dedicadas à promoção da saúde, o excesso de peso e o sedentarismo- (dois importantes fatores de risco para o desenvolvimento e mau controle metabólico do DM2), estiveram presentes de forma expressiva na população estudada.

Neste estudo, o excesso de peso esteve estatisticamente associado ao surgimento da SM $(p=0,001)$. Corroborando com esse dado, pesquisadores brasileiros, em estudo de prevalência de SM em pessoas com diabetes, revelaram que $80,6 \%$ das pessoas com a síndrome tinham excesso de peso. ${ }^{26}$

Quanto ao sedentarismo, uma pesquisa desenvolvida no estado de São Paulo buscou a relação entre a SM e a prática de atividade física, revelando que quanto maior o sedentarismo, maiores as chances para o aparecimento de SM. Igualmente, os pesquisadores também mostraram que os indivíduos sedentários com um ou mais componentes da SM apresentam doenças crônicas associadas, tais como hipercolesterolemia e diabetes. ${ }^{27}$ Vale considerar que o sedentarismo, o sobrepeso e a obesidade são condições que potencializam as alterações cardiometabólicas e promovem o aumento da CC, fator este condicionante para o aparecimento da SM, conforme a IDF., ${ }^{5,15-20}$ 
Os valores elevados neste estudo para a CC $(62,0 \%)$ e a GJ $(58,5 \%)$ estiveram bem acima dos valores médios (48,67\% e 19,8\%, respectivamente) dispostos em outras investigações de prevalência que abordavam a temática. ${ }^{24,17}$ Quanto aos valores de TG, 55,8\% dos participantes apresentaram números elevados deste marcador, discrepante dos percentuais de outros inquéritos. ${ }^{15,17}$ Nos Estados Unidos, país com elevado número de obesos, aponta-se uma alta prevalência de obesidade abdominal reportada em pesquisa que investigou a ocorrência da SM. ${ }^{16}$ No Brasil, os valores de obesidade abdominal são crescentes, e tem relação com as manifestas transformações sociodemográficas que o país enfrenta.

No que se refere ao HDL-c, esse foi, isoladamente, o componente da SM mais prevalente em diferentes estudos conduzidos na América Latina. Autores da área relatam que os níveis controlados do HDL-c são mais evidentes quando os pacientes com DM2 possuem um melhor controle glicêmico. ${ }^{1,12-14,17}$ Outrossim, considera-se mais difícil a obtenção de bons índices de HDL-c em pacientes acima dos 45 anos, uma vez que, para o alcance do controle desse tipo de colesterol, são necessárias, além de atitudes voltadas à prática de exercícios físicos regulares e alimentação saudável, uma simetria hormonal e cardiometabólica. ${ }^{1,28-29}$

Outro fator a ser considerado quanto ao incremento no diagnóstico da SM é a elevação da PA ou mesmo a presença do diagnóstico de hipertensão arterial sistêmica, presente em 49,8\% e 70,8\% da amostra estudada, respectivamente. Em uma revisão sistemática da literatura, cujo objetivo foi identificar a prevalência da SM de acordo com os critérios disponíveis, os autores constataram que a prevalência média ponderada da hipertensão foi de $52,5 \%$, bem abaixo dos valores encontrados neste estudo. Alguns autores trazem enfatizam que, quando os indivíduos com DM2 apresentam hipertensão e $\mathrm{SM}$, eles têm elevadas chances para complicações cardiovasculares severas. ${ }^{1,23}$

Referidos achados tornam-se importantes ao levar em consideração que a hipertensão é a principal comorbidade associada ao DM2, pois, de modo geral, recomenda-se a intensificação no controle pressórico, por meio, principalmente, do uso de medicações anti-hipertensivas, que apresentam impacto positivo sobre o controle metabólico, além, claro, das medidas de mudanças no estilo de vida para essa população, minimizando a possibilidade do aparecimento de doenças coronarianas. Uma vez identificada essa e ou outra comorbidade, os profissionais de saúde devem lançar mão de cuidados que contemplem os distintos componentes para o diagnóstico da SM, concentrando medidas interventivas eficazes frente ao descompasso causado pela síndrome. ${ }^{30}$

Igualmente, medidas de incentivo ao abandono do uso do cigarro e do álcool devem ser implementadas, pois além dos agravos já conhecidos, essas drogas aumentam, também, a chance do desenvolvimento da SM, uma vez que aumentam a concentração de gordura abdominal, reduzindo a sensibilidade insulínica e elevando os níveis glicêmicos, exemplificado pelos participantes desta pesquisa que, por sua vez, apresentaram alterações significativas nas variáveis metabólicas investigadas. ${ }^{26}$ Por fim, merece ser destacada a real necessidade de se desenvolver e institucionalizar ações que promovam a saúde desses pacientes. Sendo assim, ao levar em consideração que promover saúde é capacitar os indivíduos para se tornarem responsáveis e autônomos de sua própria saúde, os autores deste trabalho acreditam que a educação em saúde pode ser um instrumento valioso para esse fim, principalmente para o profissional de enfermagem, possuindo na natureza de seu trabalho, um elo entre a pessoa com a doença crônica, sua família e o serviço de saúde, tendo o desafio de auxiliar e manejar cuidados para a modificação do estilo de vida, ou mesmo, da própria enfermidade. ${ }^{31}$

Sabe-se que a SM pode ser identificada diariamente nos distintos cenários de atuação dos enfermeiros, principalmente na atenção básica de saúde, sendo esta reorientada e operacionalizada pela Estratégia Saúde da Família. Os enfermeiros dispõem de um vasto e robusto corpo de conhecimentos científicos que, a cada dia, vem sendo fortalecido.

Conhecer a SM, seus critérios diagnósticos e seus componentes, assim como os protocolos de atendimento aos pacientes com DM2 e as ferramentas de trabalho próprias da enfermagem se fazem de suma importância, uma vez que se busca e se tem como objetivo comum melhorar a qualidade de vida das pessoas com doenças crônicas.

Ao mesmo tempo, esta pesquisa tem como avanço para a qualificação da assistência de enfermagem, o norteamento para a criação de novos diagnósticos, bem como a edificação para novos fatores dispostos em taxonomias de enfermagem com menção a SM. Além disso, a individualização dos marcadores para a SM traz evidências no que tange ao risco de desenvolvimento de DCV e serve de subsídio para o estabelecimento de intervenções robustas voltadas para o empoderamento dessa população.

As limitações dessa investigação podem ser retratadas, inicialmente, por se tratar de um estudo com corte transversal e por ter usado apenas o 
critério da IDF para a detecção da SM. Além disso, para algumas variáveis, o tamanho da amostra foi reduzido, uma vez que alguns participantes se recusaram à coleta sanguínea.

\section{CONCLUSÃO}

Foi alta a prevalência da SM na população de diabéticos estudada (46,3\%). Vale ressaltar que esse número pode aumentar, uma vez que $62,0 \%$ deles já possuem o componente inicial da SM, que é a alteração na CC. Atenção especial deve ser dada às mulheres e às pessoas com excesso de peso, pois além de apresentarem percentuais significativamente maiores de SM, também apresentaram maiores alterações nos principais componentes, como CC, triglicerídeos, HDL-c e pressão arterial.

Salienta-se que estudos de prevalência da SM devam ser realizados, com distintos públicos, utilizando outros critérios diagnósticos, com amostras maiores e diferentes desenhos metodológicos. Assim, a comunidade científica brasileira possuirá dados, cada vez mais robustos, que possam retratar fielmente a problemática da SM no país e outras populações.

\section{AGRADECIMENTOS}

À Fundação de Amparo a Pesquisa do Estado do Piauí (FAPEPI).

\section{REFERÊNCIAS}

1. Vidigal FC, Bressan J, Babio N, Salas-Salvadó J. Prevalence of metabolic syndrome in Brazilian adults: a systematic review. BMC Public Health [Internet]. 2013 [cited 2016 Jun10]; 13:1198. Available from: http:// www.ncbi.nlm.nih.gov/pmc/articles/PMC3878341/ pdf/1471-2458-13-1198.pdf

2. Araújo MFM, Freitas RWJF, Lima ACS, Pereira DCR, Zanetti ML, Damasceno MMC. Relation between sleep quality and metabolic syndrome among university students. Texto Contexto Enferm [Internet]. 2015 [cited 2016 Jun10]; 24(2):505-12. Available from: http:/ / www.scielo. br/pdf/tce/v24n2/pt_0104-0707-tce-24-02-00505.pdf

3. Pinzón JB, Serrano NC, Díaz LA, Mantilla G, Velasco HM, Martínez LX, et al. Impact of the new definitions in the prevalence of the metabolic syndrome in an adult population at Bucaramanga, Colombia. Biomédica (Bogotá) [Internet]. 2007 [cited 2016 Jun10]; 27(2):172-9. Available from: http://www.scielo.org.co/pdf/bio/v27n2/v27n2a04.pdf

4. Mujica V, Leiva E, Icaza G, Diaz N, Arredondo M, Moore-Carrasco R, et al. Evaluation of metabolic syndrome in adults of Talca city, Chile. NutrJ (Online) [Internet]. 2008 [cited 2016 Jun15]; 7:14. Available from: http:// www.ncbi.nlm.nih.gov/pmc/articles/PMC2397433/ pdf/1475-2891-7-14.pdf

5. Lira Neto JCG, Xavier MA, Borges JWP, Araújo MFM, Damasceno MMC, Freitas RWJF. Prevalence of meta- bolic syndrome in individuals with type 2 diabetes mellitus. Rev Bras Enferm [Internet]. 2017 [cited 2017 Jul 24]; 70(2):265-70. Available from: http://www.scielo.br/ pdf/reben/v70n2/pt_0034-7167-reben-70-02-0265.pdf

6. Saboya PP, Bodanese LC, Zimmermann PR, Gustavo AS, Macagnan FE, Feoli AP, Oliveira MS. Lifestyle intervention on metabolic syndrome and its impact on quality of life: a randomized controlled trial. Arq Bras Cardiol [Internet]. 2017 [cited 2017 Jul 24]; 108(1):60-9. Available from: http:/ / www.scielo.br/pdf/abc/v108n1/pt_0066782X-abc-20160186.pdf

7. Lira Neto JCG, Silva AP, Araújo MFM, Damasceno MMC, Landim MBP, Freitas RWJF. Metabolic control and medication adherence in people with diabetes mellitus. Acta Paul Enferm [Internet]. 2017 [cited 2017 Jul 24]; 30(2):152-8. Available from: http:/ /www. scielo.br/scielo.php?script=sci_arttext $\&$ pid=S010321002017000200152\&lng=pt.

8. Oliveira GF, Oliveira TR, Rodrigues FF, Corrêa LF, Arruda TB, Casulari LA. Prevalence of metabolic syndrome in the indigenous population, aged 19 to 69 years, from Jaguapiru Village, Dourados (MS), Brazil. EthnDis. 2011; 21(3):301-6.

9. Gronner MF, Bosi PL, Carvalho AM, Casale G, Contrera D, Pereira MA, et al. Prevalence of metabolic syndrome and its association with educational inequalities among Brazilian adults: a population-based study. Braz J Med Biol Res [Internet]. 2011 [cited 2016 Jun 15]; 44(7):7139. Available from: http://www.scielo.br/pdf/bjmbr/ v44n7/997.pdf

10.Fonseca MJ, Gaio R, Lopes C, Santos AC. Association between dietary patterns and metabolic syndrome in a sample of Portuguese adults. Nutr j (Online) [Internet]. 2012 [cited 2016 Jun 15]; 11: 64. Available from: http:/ / www.ncbi.nlm.nih.gov/pmc/articles/PMC3493346/ pdf/1475-2891-11-64.pdf

11.Corbatón-Anchuelo A, Martínez-Larrad MT, FernándezPérez C, Vega-Quiroga S, Ibarra-Rueda JM, Serrano-Ríos M. Metabolic syndrome, adiponectin, and cardiovascular risk in Spain (the Segovia study): impact of consensus societies criteria. MetabSyndrRelatDisord. 2013; 11(5):309-18.

12.Wagner A, Dallongeville J, Haas B, Ruidavets JB, Amouyel P, Ferrières J, et al. Sedentary behaviour, physical activity and dietary patterns are independently associated with the metabolic syndrome. Diabetes Metab [Internet]. 2012 [cited 2016 Jun15]; 38(5):428-35. Available from: http://www.diabet-metabolism.com/ article/S1262-3636(12)00081-X/pdf

13. Beltrán-Sánchez H, Harhay MO, Harhay MM, McElligott S. Prevalence and trends of metabolic syndrome in the adult U.S. population, 1999-2010. J Am CollCardiol. [Internet]. 2013 [cited 2016 Jun15]; 62(8):697-703. Available from: http://www.ncbi.nlm.nih.gov/pmc/ articles/PMC3756561/pdf/nihms501854.pdf

14.Belfki H, Ali SB, Aounallah-Skhiri H, Traissac P, Bougatef S, Maire B, et al. Prevalence and determinants of the metabolic syndrome among Tunisian adults: results of the transition and health impact in North Africa (TAHINA) project. Public Health Nutr [Internet]. 2012 [cited 2016 Jun15]; 16(4): 582-90. Available from: http://journals.cambridge.org/download.php?file $=\% 2 \mathrm{FPHN} \% 2 \mathrm{~F}$ PHN16_04\%2FS1368980012003291a.pdf\&code=b156769 3dadcd944e332fec41a70bb64 
15.Isordia-Salas I, Santiago-German D, Rodriguez-Navarro H, Almaraz-Delgado M, Leanos-Miranda A, Anaya-Gomez F, etal. Prevalence of metabolic syndrome components in an urban mexican sample: comparison between two classifications. Exp Diabetes Res [Internet] 2012 [cited 2017 Jul 25]; 2012:202540. Available from: https:/ / www.ncbi.nlm.nih.gov/pmc/articles/PMC3235771/ pdf/EDR2012-202540.pdf

16.Khan SA, Jackson RT. The prevalence of metabolic syndrome among low-income south Asian Americans. Public Health Nutr [Internet] 2016 [cited 2017 Jul 25];19(3):418-28. Available from: https://www.cambridge.org/core/services/aop-cambridge-core/content/view / D396499EC031F3AA4BEEE4688E993141/ S1368980015001330a.pdf/prevalence_of_metabolic_syndrome_among_lowincome_south_asian_americans.pdf

17.Davila EP, Quintero MA, Orrego ML, Ford ES, Walke H, Arenas MM, etal. Prevalence and risk factors for metabolic syndrome in Medellin and surrounding municipalities, Colombia, 2008-2010. PrevMed. 2013;56(1):30- 4.

18.Szigethy E, Szeles G, Horvath A, Hidvegi T, Jermendy G, Paragh G,etal. Epidemiology of the metabolic syndrome in Hungary. Public Health [Internet] 2012 [cited 2017 Jul 25];126(2):143-9. Available from: http://www.publichealthjirnl.com/article/S0033-3506(11)00339-8/pdf

19.Amirkalali B, Fakhrzadeh H, Sharifi F, Kelishadi R, Zamani F, Asayesh H, Safiri S, Samavat T, Qorbani M. Prevalence of metabolic syndrome and its components in the iranian adult population: a systematic review and metaanalysis. Iran Red Crescent Med J [Internet] 2015 [cited 2017 Jul 25];17(12):e24723. Available from: https:// www.ncbi.nlm.nih.gov/pmc/articles/PMC4706734/ pdf/ircmj-17-12-24723.pdf

20.Dutra ES, Carvalho KMB, Miyazaki E, Hamann EM, Kiyomi Ito M. Metabolic syndrome in central Brazil: prevalence and correlates in the adult population. Diabetol. MetabSyndr[Internet] 2012 [cited 2017 Jul 24];4(1):20. Available from: https://www.ncbi.nlm.nih.gov/pmc/ articles/PMC3457864/pdf/1758-5996-4-20.pdf

21.Arthur FKN, Michael AF, Osei-Yeboah J, Mensah FO, Owusu L.The prevalence of metabolic syndrome and its predominant components among pre-and postmenopausal Ghanaian women. BMC Res Notes [Internet].2013 [cited 2017 Jul 24]; 6:446. Available from: https:// www.ncbi.nlm.nih.gov/pmc/articles/PMC3843598/ pdf/1756-0500-6-446.pdf

22.Albuquerque GA, Leite MF, Belém JM, Nunes JFC, Oliveira MA, Adami F. The man in primary healthcare: perceptions of nurses about the implications of gender in health. Esc Anna Nery [Internet]. 2014 [cited 2016 Jun 20]; 18(4):607-14. Available from: http://www.scielo. br/pdf/ean/v18n4/1414-8145-ean-18-04-0607.pdf

23. Bhatti GK, Bhadada SK, Vijayvergiya R, Mastana SS, Bhatti JS. Metabolic syndrome and risk of major coro- nary events among the urban diabetic patients: North Indian Diabetes and Cardiovascular Disease Study-NIDCVD-2. J Diabetes Its Complicat. [Internet]. 2016 [cited 2017 Jul 24]; 30(1):72-8. Available from: https:/ /www. ncbi.nlm.nih.gov/pubmed/26271411

24.Nsiah K, Shang VO, Boateng KA, Mensah FO. Prevalence of metabolic syndrome in type 2 diabetes mellitus patients. Int J Appl Basic Med Res [Internet]. 2015 [cited 2017 Jul 24]; 5(2):133-8. Available from: https://www. ncbi.nlm.nih.gov/pmc/articles/PMC4456889/

25.Paula JAT, Moreira OC, Silva CD, Silva DS, Amorim PRS. Metabolic syndrome prevalence in elderly of urban and rural communities participants in the HIPERDIA in the city of Coimbra/MG, Brazil. Invest Educ Enferm. [Internet] 2015 [cited 2017 Jul 24]; 33(2):325-33. Available from: http://www.scielo.org.co/pdf/iee/v33n2/ v33n2a15.pdf

26. Maccaronea SD; Lima DB; Ferreira EB. Rastreamento da síndrome metabólica e qualidade de vida dos diabéticos adscritos a uma unidade de estratégia de saúde da família em um município do sul de Minas Gerais. Rev Aten Saúde [Internet]. 2017 [cited 2017 Jul 25]; 15(51):98-105. Available from: http://seer.uscs.edu.br/index.php/revista_ciencias_saude/article/view/4370/pdf.

27.Turi BC, Codogno JS, Fernandes RA, Monteiro HL. Low levels of physical activity and metabolic syndrome: cross-sectional study in the Brazilian public health system. Ciênc Saúde Coletiva [Internet] 2016 [cited 2017Jul 25]; 21(4):1043-50.Availablefrom: http://www.scielo. $\mathrm{br} / \mathrm{pdf} / \mathrm{csc} / \mathrm{v} 21 \mathrm{n} 4 / 1413-8123-\mathrm{csc}-21-04-1043 . p d f$

28.Wang GR, Li L, Pan YH, Tian GD, Lin WL, Li Z, et al. Prevalence of metabolic syndrome among urban community residents in China. BMC Public Health [Internet]. 2013 [cited 2016 Jun 20]; 13:599. Available from: http:/ / www.ncbi.nlm.nih.gov/pmc/articles/PMC3734094/ pdf/1471-2458-13-599.pdf

29. Gomes-Villas Boas LC, Foss-Freitas MC, Pace AE. Adherence of people with type 2 diabetes mellitus to drug treatment. Rev Bras Enferm [Internet]. 2014 [cited 2016 Jun 20]; 67(2):268-73. Available from: http:/ / www.scielo.br/pdf/ reben/v67n2/0034-7167-reben-67-02-0268.pdf.

30.Lima DBS, Moreira TMM, Borges JWP, Rodrigues MTP. Association between treatment compliance and different types of cardiovascular complications in arterial hypertension patients. Texto Contexto Enferm [Internet]. 2016 [cited 2017 Jul 24]; 25(3):e0560015. Available from: http:/ / www.scielo.br/scielo.php?script=sci_arttex t\&pid=S0104-07072016000300302\&lng=en

31.Soratto J, Pires DEP, Dornelles S, Lorenzetti J. Family health strategy: a technological innovation in health. Texto Contexto Enferm [Internet]. 2015 [cited 2017 Jul 26]; 24(2):584-92. Available from: http://www. scielo.br/scielo.php?script=sci_arttext\&pid=S010407072015000200584\&lng=en
Correspondência: José Cláudio Garcia Lira Neto

Universidade Federal do Piauí, Departamento de Enfermagem Campus Amílcar Ferreira Sobral

64800-000 - BR 343, km 3,5 Meladão, Floriano, PI, Brasil

E-mail: jclira@live.com
Recebido: 04 de outubro de 2016 Aprovado: 11 de outubro de 2017

This is an Open Access article distributed under the terms of the Creative Commons (CC BY). 